\title{
A Hemostatic Technique in Robot-Assisted Laparoscopic Partial Nephrectomy and Its Impact on Renal Function
}

\author{
Deepak Raghavan $^{1}$, Mathisekaran Thangarasu ${ }^{1}$, Sanjay Prakash J ${ }^{1}$, Rajesh Paul ${ }^{1}$, Nivash Selvaraj ${ }^{1}$ \\ 1. Urology, Apollo Hospitals, Chennai, IND
}

Corresponding author: Mathisekaran Thangarasu, mathisekaran@gmail.com

\section{Abstract \\ Purpose}

Robot-assisted partial nephrectomy (RAPN) has become popular in recent years for small renal masses. We describe a technique of suturing renal defects during RAPN that is reliable and quick, does not necessitate the need for hemostatic agents, and reduces perioperative complications.

\section{Materials and methods}

A total of 24 patients who underwent RAPN were included in the study period between 2013 and 2018 and data were analyzed. Perioperative and postoperative outcomes were measured and compared.

\section{Results}

The median tumor size was $4 \mathrm{~cm}$. Median warm ischemia time was 41 minutes (IQR: $38-45$ minutes) and estimated blood loss was $150 \mathrm{~mL}$ (IQR: 120-200 mL). There were no major intraoperative complications or conversions to open surgery. No urine leaks or postoperative bleedings were observed.

\section{Conclusion}

Our technique is safe and effective. It negates the use of hemostatic agents, decreases perioperative complications, and negates that determination of long-term renal function is not associated with prolonged warm ischemia time alone. Hence, we propose that our technique is safe in partial nephrectomy when the pelvic calyceal system and renal vessels are opened in multiple locations.

Review began 05/05/2021 Review ended 05/12/2021 Published 05/19/2021

\section{○ Copyright 2021}

Raghavan et al. This is an open access article distributed under the terms of the Creative Commons Attribution License CC-BY 4.0, which permits unrestricted use, distribution, and reproduction in any medium, provided the original author and source are credited.

Categories: Urology, General Surgery, Nephrology

Keywords: partial nephrectomy, tumor, hemostatic, complications, renal function

\section{Introduction}

Renal cell carcinoma is the commonest cancer seen in the genitourinary department. With the increased incidence of diagnosing small renal masses, urologists are motivated to improve the contemporary techniques [1]. Currently, small masses of kidneys are well managed by partial nephrectomy (PN) as a substitute for radical nephrectomy. Presently, robot-assisted partial nephrectomy (RAPN) is becoming the standard of care for renal masses, which has its own advantages such as 3D vision, better ergonomics, lower morbidity, reduced postoperative complications, and shorter hospital stay. However, the renal defect closure and control of bleeding under pressure of a ticking clock remains a common problem. We describe a technique in RAPN which minimizes the devascularization of parenchyma without any need of hemostatic agents and preserves the maximum healthy renal parenchyma and fewer complications.

\section{Materials And Methods}

This study was approved by the Institutional Ethics Committee - Clinical Studies, Apollo Hospitals, Chennai, India, and was conducted between January 2013 and March 2018. The written informed consent was obtained from the patients prior to the procedure. A total of 24 patients underwent RAPN. All the procedures were performed by a single experienced robotic surgeon. All the patients underwent preoperative workup, which included serum creatinine, estimated glomerular filtration rate (eGFR), and radiological assessment by contrast-enhanced triphasic CT for nephrometric score. The measured outcomes were operative time, warm ischemia time (WIT), estimated blood loss, margin status, and postoperative complications. The patients were followed up for 24 months postoperatively for the renal function and cancer status. Serum creatinine and eGFR were used to assess the renal function during the follow-up period. The Modification of Diet in Renal Disease (MDRD) study equation was used to assess the renal status of the patient [2].

\section{Statistical analysis}




\section{Cureus}

The data were statistically analyzed using SPSS 20.0 Version (IBM Corp., Armonk, NY). The non-normally distributed variables are presented as the median. Nominal data are presented as a number or percentage and compared using the chi-square test. Differences were compared between the follow-up period and the baseline. A p-value of less than 0.05 was considered statistically significant.

\section{Surgical technique}

Under general anesthesia and antibiotic coverage, the patient was in lateral decubitus position with parts painted and draped. No prior double J stenting was performed in any patients. Pneumoperitoneum was created, and the standard five- or six-port insertion technique was followed (1-12 mm or 4-8 mm based on the side). The DaVinci Xi Surgical System (Intuitive Surgical Inc., Sunnyvale, CA) was docked. The following steps were involved:

Step 1: The colon was mobilized and dropped down.

Step 2: The gonadal vein and ureter bundle were identified and independently preserved, and dissection proceeded towards the hilum.

Step 3: The hilar vessels were skeletonized, the renal artery as well as the renal vein were looped, and dissection was performed in extra gerota's fascia.

Step 4: Gerota's fascia overlying the tumor was opened and the tumor was visualized. Intra-operative ultrasound was used to ascertain tumor margins and depth.

Step 5: Renal arteries were clamped with vascular Scanlan ${ }^{\circledR}$ Bulldog Clamps (Scanlan International, Saint Paul, MN).

Step 6: Scoring of the tumor was done, and the tumor was dissected with healthy tissue margin (Figure 1).

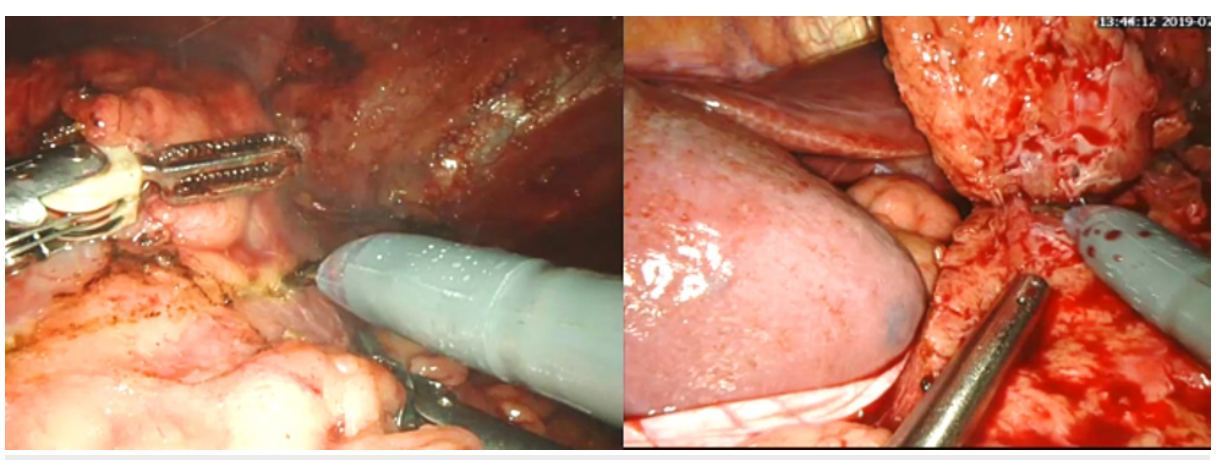

FIGURE 1: Tumor scoring and excision

In the next step (step 7), the exposed individual vessels were ligated at the resected renal parenchyma and tumor base with 4-0 Vicryl suture (Ethicon, Somerville, NJ) using a figure-of-eight technique (Figure 2). If the pelvic calyceal system (PCS) was opened, it was meticulously closed with 4-0 Vicryl suture in a continuous manner. The cortex and corticomedullary junction were closed with continuous sutures as an inner layer renorrhaphy with 2-0 Stratafix ${ }^{\mathrm{TM}}$ (Ethicon). Interrupted sliding clip outer layer renorrhaphy was performed with 1-0 Vicryl suture (Figure 3). Finally, vascular clamps were released and a flat drain was placed. The specimen was bagged and retrieved via incision in the iliac fossa. Wound was closed in layers, and skin was sutured with 3-0 Monocryl suture (Ethicon). 


\section{Cureus}

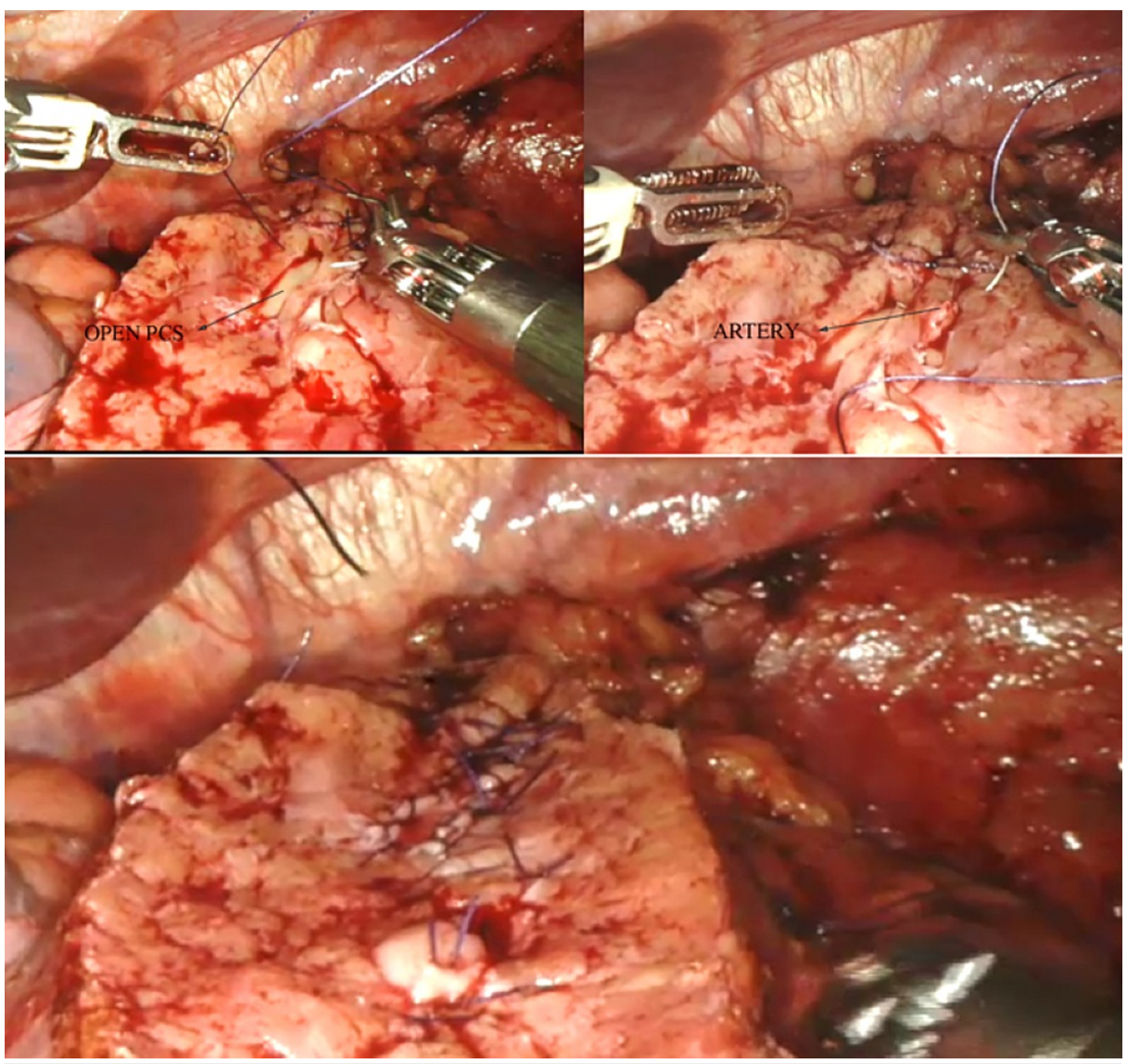

FIGURE 2: Completion of individual renal vessels ligation and closure of the opened pelvic calyceal system

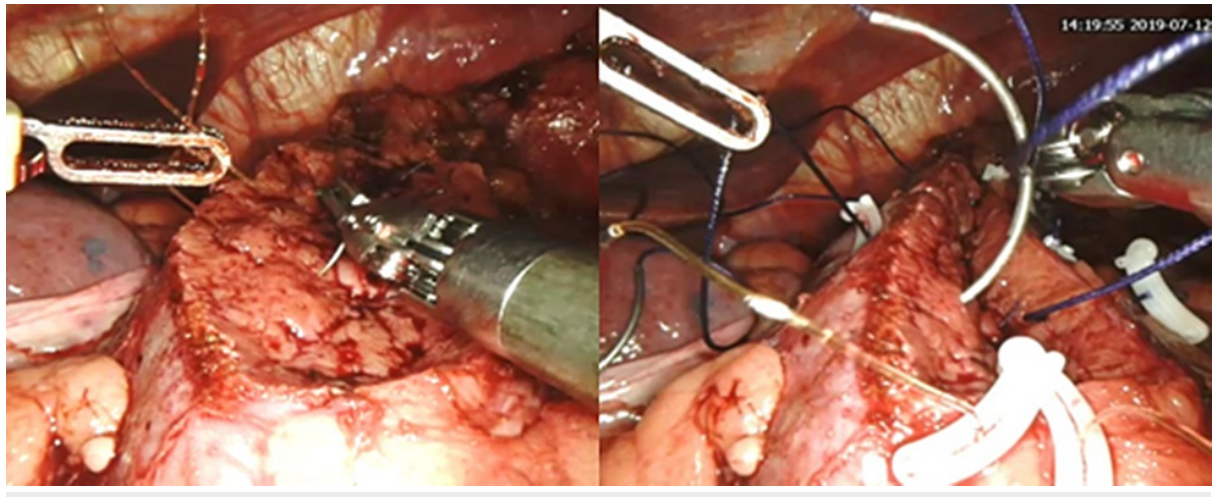

FIGURE 3: Completion of the inner corticomedullary junction by continuous suture and outer renorraphy with interrupted layer

\section{Results}

All the patients successfully completed the study. The median age of the patient was 50 years. Preoperatively four patients were having chronic kidney disease (CKD) stage $\geqslant 3$ disease. The median preoperative creatinine and eGFR were $0.95 \mathrm{mg} / \mathrm{dL}$ and $90 \mathrm{~mL} / \mathrm{min} / 1.73 \mathrm{~m}^{2}$, respectively. The demographic profile of the patient is given in Table 1 . 


\section{Cureus}

\section{Characteristics}

Age (in years), median (IQR)

50 (17-59)

Gender \% (male/female)

$20(83 \%) / 4(17 \%)$

BMI, median (IQR)

$24.7(22.9-27.1)$

ASA grade (1/2/3/4)

$2 / 6 / 14 / 2$

CKD stage $\geq 3(\%)^{*}$

$4(17 \%)$

Preoperative creatinine ( $\mathrm{mg} / \mathrm{dL}$ ), median (IQR)

$0.95(0.8-1.2)$

Preoperative eGFR (mL/min/1.73m²), median (IQR)

$90(66-101)$

Laterality (right/left), \%

Clinical stage

T1a, $\mathbf{n}$

T1b, $n$

Site

Upper, $\mathrm{n}$

8

Interpole, $n$

Lower, $\mathrm{n}$

Renal score, median (IQR)

\section{TABLE 1: Demographic profile of the patient $(n=24)$}

ASA, American Society of Anesthesiologists; BMI, body mass index; CKD, chronic kidney disease; eGFR, estimated glomerular filtration rate; IQR, interquartile range

${ }^{*}$ eGFR $<60 \mathrm{~mL} / \mathrm{min} / 1.73 \mathrm{~m}^{2}$

There was no conversion to open surgery. The median operative time was 130 minutes (IQR: 108-160 minutes), and the median WIT was 41 minutes (IQR: 38-45 minutes) and for this step it was 3 minutes. There were no urine leak or major intraoperative and postoperative complications recorded in our study. The median estimated blood loss was $150 \mathrm{~mL}$ (IQR: $120-200 \mathrm{~mL}$ ), and the median length of stay was four days. All patients were having zero positive margins. The overall perioperative outcomes are shown in Table 2 . 


\section{Cureus}

\section{Variables}

Operative time (minutes)

Estimated blood loss ( $\mathrm{mL}$ )

Warm ischemic time (minutes)

Margin positivity

Pathological diagnosis

Benign

Malignant

Length of stay (days)

\section{Median (IQR)}

130 (108-160)

$150(120-200)$

$41(38-45)$

Nil

2

22

$4(3-5)$

TABLE 2: Overall perioperative outcome

IQR, interquartile range

Moreover, the median serum creatinine levels were significantly increased and eGFR was significantly decreased after RAPN. However, no significant differences were observed between the preoperative values after one-year and two-year follow-up. Tables 3, 4 show information about the renal function changes over time.

\begin{tabular}{|l|l|l|l|l|l|l|l|l|}
\hline Parameters & Preoperative value & Day 2 & Three months & Six months & P1 & P2 & P3 \\
\hline Creatinine, median (IQR) & $0.9(0.8-1.2)$ & $1.5(1.3-1.5)$ & $2(1.3-2.6)$ & $1.5(1.2-2.2)$ & 0.01 & 0.014 & 0.02 \\
\hline eGFR (mL/min/1.73 $\left.{ }^{2}\right)$, median (IQR) & $90(67-102)$ & $49(36-63)$ & $37(30-57)$ & $50(29-68)$ & 0.002 & 0.01 & 0.04 \\
\hline
\end{tabular}

\section{TABLE 3: Renal function changes over time}

P1: statistical difference between preoperative creatinine value and day 2 creatinine value.

P2: statistical difference between preoperative creatinine value and three-month creatinine value.

P3: statistical difference between preoperative creatinine value and six-month creatinine value.

eGFR, estimated glomerular filtration rate; IQR, interquartile range

\begin{tabular}{|c|c|c|c|c|c|}
\hline Parameters & Preoperative value & One year & Two years & P1 & P2 \\
\hline Creatinine, median (IQR) & $0.9(0.8-1.2)$ & $1.2(1.1-1.5)$ & $1.2(1.1-1.5)$ & 0.39 & 0.65 \\
\hline eGFR $\left(\mathrm{mL} / \mathrm{min} / 1.73 \mathrm{~m}^{2}\right)$, median (IQR) & $90(67-102)$ & $63(45-73)$ & $61(50-78)$ & 0.47 & 0.32 \\
\hline \multicolumn{6}{|c|}{ TABLE 4: Renal function changes over time } \\
\hline \multicolumn{6}{|c|}{ P1: statistical difference between preoperative creatinine value and one-year creatinine value. } \\
\hline \multicolumn{6}{|c|}{ P2: statistical difference between preoperative creatinine value and two-year creatinine value } \\
\hline eGFR, estimated glomerular filtration rate; & ile range & & & & \\
\hline
\end{tabular}

\section{Discussion}

RARP is the standard of care for most of the patients with T1 renal lesions in many tertiary centers. The 
renal function preservation is the primary aim in PN and can be done by open, laparoscopic, or robotic methods [3]. The various factors that predict renal functional status after PN include tumor, patient, and surgery-related factors, out of which the only factor that can be optimized is the surgery-related factor [4].

The clamping of renal vessels in PNs is generally needed to manage blood loss, attain a negative-positive margin, and execute precise renorrhaphy. Numerous techniques have been documented in the literature with the aim of preserving renal parenchyma and have fewer complications [5-7]. The present study describes a surgical technique in which the renal vasculature is preserved and parenchymal devascularization is minimized with no major postoperative complications.

In the present study, the median warm ischemic time was 41 minutes (IQR: 38-45 minutes). This was in contrast with many other studies. However, several studies have shown that the final renal function of the kidney subjected to PN correlated primarily with the preserved parenchyma volume, whereas only a secondary role was played by WIT, irrespective of whether WIT was achieved by flow restriction or hypothermia [8]. Recently, Nahar et al. reported that in PN, baseline renal function and the amount of preserved parenchyma play a major role in determining the final outcome. The limited duration of ischemia does not have a significant role impact on final renal function [9]. Moreover, the focus should rather be on appropriate perioperative management of patients, which includes controlling diabetes and hypertension, identifying proteinuria and $\mathrm{CKD}$, preservation of renal parenchyma volume, and timely referral to nephrologists for optimization postoperatively. In our study, even though the serum creatinine was significantly increased and eGFR was significantly decreased, at two-year follow-up, we did not encounter deterioration in renal function.

In our study, the technique of independently suturing opened blood vessels and the opened PCS with Vicryl before performing the two-layer renorrhaphy resulted in a median blood loss of $150 \mathrm{~mL}$. No significant bleeding was encountered upon clamp release intraoperatively or postoperatively. In addition, Seideman et al. showed reduced incidence of major intraoperative bleeding by using barbed suture [10]. Similar outcomes were documented in other series using V-Loc ${ }^{\text {TM }}$ (Medtronic, Minneapolis, MN) [11-12]. The results of the present study were in concordance with the previous studies.

Omae et al. reported an unanticipated increasing rate of about $21.7 \%$ for unruptured asymptomatic pseudoaneurysm of the renal artery, as identified by CT arteriography [13]. In addition, Jain et al. in a systematic and comparative analysis reported symptomatic pseudoaneurysm in $1.96 \%$ of cases [14]. In this present study, we did not encounter any such incident during the postoperative follow-up. One of the main limitations of this study is the lack of a control group and small sample size with low complexity.

\section{Conclusions}

Thus, our technique is simple and effective in reducing perioperative complications and dealing with difficult anatomy. It also negates the use of hemostatic agents and that determination of long-term renal function is not associated with prolonged WIT alone. Hence, we propose that our technique is safe in PN when PCS and renal vessels are opened in multiple locations. However, further randomized control studies with lengthier follow-up are needed.

\section{Additional Information \\ Disclosures}

Human subjects: Consent was obtained or waived by all participants in this study. Institutional Ethics Committee - Clinical Studies, Apollo Hospitals, Chennai issued approval ECR/37/Inst/TN/2013/RR-15. Animal subjects: All authors have confirmed that this study did not involve animal subjects or tissue. Conflicts of interest: In compliance with the ICMJE uniform disclosure form, all authors declare the following: Payment/services info: All authors have declared that no financial support was received from any organization for the submitted work. Financial relationships: All authors have declared that they have no financial relationships at present or within the previous three years with any organizations that might have an interest in the submitted work. Other relationships: All authors have declared that there are no other relationships or activities that could appear to have influenced the submitted work.

\section{References}

1. Jemal A, Bray F, Center MM, Ferlay J, Ward E, Forman D: Global cancer statistics. CA Cancer J Clin. 2011, 61:69-90. 10.3322/caac.20107

2. Levey AS, Bosch JP, Lewis JB, Greene T, Rogers N, Roth D: A more accurate method to estimate glomerular filtration rate from serum creatinine: a new prediction equation. Modification of Diet in Renal Disease Study Group. Ann Intern Med. 1999, 130:461-70. 10.7326/0003-4819-130-6-199903160-00002

3. Ljungberg B, Bensalah K, Bex A, et al.: EAU Guidelines on Renal Cell Carcinoma . EAU Guidelines Office, Arnhem, The Netherlands; 2021.

4. Lane BR, Babineau DC, Poggio ED, Weight CJ, Larson BT, Gill IS, Novick AC: Factors predicting renal functional outcome after partial nephrectomy. J Urol. 2008, 180:2363-8; discussion 2368-9. 10.1016/j.juro.2008.08.036 


\section{Cureus}

5. Antonelli A, Veccia A, Francavilla S, et al.: On-clamp versus off-clamp robotic partial nephrectomy: a systematic review and meta-analysis. Urologia. 2019, 86:52-6. 10.1177/0391560319847847

6. Minervini A, Campi R, Sessa F, et al.: Positive surgical margins and local recurrence after simple enucleation and standard partial nephrectomy for malignant renal tumors: systematic review of the literature and metaanalysis of prevalence. Minerva Urol Nefrol. 2017, 69:523-38. 10.23736/S0393-2249.17.02864-8

7. Xu C, Lin C, Xu Z, Feng S, Zheng Y: Tumor enucleation vs. partial nephrectomy for T1 renal cell carcinoma: a systematic review and meta-analysis. Front Oncol. 2019, 9:473. 10.3389/fonc.2019.00473

8. Mir MC, Campbell RA, Sharma N, et al.: Parenchymal volume preservation and ischemia during partial nephrectomy: functional and volumetric analysis. Urology. 2013, 82:263-8. 10.1016/j.urology.2013.03.068

9. Nahar B, Bhat A, Parekh DJ: Does every minute of renal ischemia still count in 2019? unlocking the chains of a flawed thought process over five decades. Eur Urol Focus. 2019, 5:939-42. 10.1016/j.euf.2019.03.019

10. Seideman C, Park S, Best SL, Cadeddu JA, Olweny EO: Self-retaining barbed suture for parenchymal repair during minimally invasive partial nephrectomy. J Endourol. 2011, 25:1245-7. 10.1089/end.2011.0105

11. Bezwada RS, Jamiolkowski DD, Lee IY, et al.: Monocryl suture, a new ultra-pliable absorbable monofilament suture. Biomaterials. 1995, 16:1141-8. 10.1016/0142-9612(95)93577-z

12. Weld KJ, Arzola J, Montiglio C, Bush AC, Cespedes RD: Lapra-Ty holding strength and slippage with various suture types and sizes. Urology. 2008, 71:32-5. 10.1016/j.urology.2007.08.061

13. Omae $\mathrm{K}$, Kondo $\mathrm{T}$, Takagi $\mathrm{T}$, et al.: Renal sinus exposure as an independent factor predicting asymptomatic unruptured pseudoaneurysm formation detected in the early postoperative period after minimally invasive partial nephrectomy. Int J Urol. 2015, 22:356-61. 10.1111/iju.12696

14. Jain S, Nyirenda T, Yates J, Munver R: Incidence of renal artery pseudoaneurysm following open and minimally invasive partial nephrectomy: a systematic review and comparative analysis. J Urol. 2013, 189:1643-8. 10.1016/i.juro.2012.11.170 